\title{
UMA ANÁLISE PRELIMINAR DAS CONTRIBUIÇÕES DA MODULARIDADE EM PRODUTO AO ECODESIGN
}

\section{AN PRELIMINAR ANALYSIS OF PRODUCT MODULARITY CONTRIBUITIONS TO ECODESIGN}

\author{
Flávio Issao Kubota * E-mail: flavioissao.kubota@gmail.com \\ Lucila Maria de Souza Campos * E-mail: lucila@deps.ufsc.br \\ Paulo Augusto Cauchick Miguel *E-mail: cauchick@deps.ufsc.br \\ *Universidade Federal de Santa Catarina (UFSC), Florianópolis,SC
}

Resumo: O presente trabalho tem como objetivo fazer uma análise preliminar da literatura acerca das contribuições geradas pela modularidade em produto aos princípios que orientam o ecodesign. $\mathrm{O}$ estudo considerou o fato de a modularidade ser uma crescente estratégia de desenvolvimento de produtos nas organizações, trazendo benefícios e contribuições diversas. $O$ trabalho teve a abordagem teórico-conceitual como método de pesquisa, que se iniciou com uma busca estruturada por artigos relacionados aos dois assuntos (modularidade e ecodesign), seguida de uma busca não estruturada, ambas considerando as expressões-chave relativas aos temas. Foi encontrado um número limitado de publicações abordando os dois temas, no entanto, pode-se evidenciar que a modularidade em produto traz potenciais benefícios ambientais no desenvolvimento e consumo de produtos, especialmente com produtos multifuncionais e com facilidade de reutilização de módulos, desmontagem e descarte pós-uso. Conclui-se assim que a modularidade em produto pode gerar contribuições e benefícios relevantes aos princípios e práticas do ecodesign, sendo necessárias pesquisas empíricas para posterior comparação com a literatura.

Palavras-chave: Modularidade. Modularidade em produto. Ecodesign.

Abstract: This study aims to conduct a preliminary literature analysis regarding the contributions of modularity to ecodesign principles. The research considered that modularity is a growing product development strategy in organizations, bringing a variety of contributions and benefits. A theoreticalconceptual approach was used as research method, which started with a structured search for related subject (modularity and ecodesign) articles, followed by a non-structured search, both considering the related keywords. We found a limited number of publications regarding the two topics. However we could see that product modularity brings potential environmental benefits in product development and use, mainly through multifunctional products and modules reuse, disassembly and easy disposal after using. We conclude, hence, that product modularity can provide relevant contributions and benefits to ecodesign principles and practices, being needed empirical studies for future literature comparison.

Key-words: Modularity. Product modularity. Ecodesign.

\section{INTRODUÇÃO}

O desenvolvimento de novos produtos, nas últimas décadas, tem-se tornado uma atividade desafiadora nas organizações, principalmente em setores onde a 
concorrência e a competitividade são mais intensas. Clientes mais bem informados têm feito com que as empresas busquem o aumento crescente da qualidade em seus produtos e serviços, ou seja, a busca por maior qualidade já não é mais uma questão opcional há algum tempo. Este fato tem gerado uma diferenciação de produtos e serviços no mercado, o que, simultaneamente, proporciona a melhoria contínua nas organizações, mantendo-as atuantes no mercado. Ainda, as equipes de desenvolvimento de produtos têm enfrentado diversos desafios nesse processo, uma vez que as pressões por resultados mais eficazes e redução no tempo de lançamento do produto estão cada vez mais intensas (CAUCHICK MIGUEL et al., 2009).

Além dessas preocupações e desafios, o crescimento na produção e consumo de bens e serviços é um fator inquietante e que gera degradação ambiental (BORCHARDT et al., 2009). Conforme relata Luttropp e Lagerstedt (2006), as questões ambientais precisam agora ser consideradas como parte integrante do processo global de desenvolvimento de produtos. Desse modo, conceitos como o Ecodesign, produção mais limpa e desenvolvimento de produto sustentável promovem o uso de técnicas ambientalmente amigáveis para a concepção, projeto e fabricação de produtos (BYGGETH et al., 2007; BORCHARDT et al., 2009).

Na década de 1960, a modularidade surgiu na indústria de computadores, gerando vantagem competitiva e demonstrando significativa importância no processo de desenvolvimento de produto (ARNHEITER; HARREN, 2006). Para Cardoso e Kistmann (2008), a modularidade contribui no desenvolvimento de produtos que tenham possibilidade de atender mercados diversos. Trata-se de um conceito utilizado desde o início do século XXI, sendo seu uso em projetos de produto uma tendência atual, não só em tecnologia, mas na indústria de modo geral (SILVA; CAUCHICK MIGUEL, 2006).

Alguns autores têm pesquisado o tema modularidade em produtos (CARIDI et al., 2012; HUANG et al., 2012; LAU et al., 2011), processos (PARENTE et al., 2011; JACOBS et al., 2011), em serviços (GEUM et al., 2012; LIN; PEKKARINEN, 2011; BASK et al., 2011) e/ou produção modular (RODRIGUES et al., 2009; DORAN et al., 2007), bem como seus impactos na qualidade final dos produtos (LAU et al., 2009), 
fatores críticos no gerenciamento de projetos modulares (LAU et al., 2010) e vantagens competitivas geradas com a adoção da estratégia modular (JACOBS et al., 2007). No entanto, percebe-se pela literatura que estudos relacionados aos benefícios da modularidade na perspectiva ambiental são raros, apesar de existirem publicações recentes acerca dessa relação, como os estudos de Nunes e Bennett (2008), Christensen (2011) e Huang et al. (2012). Adicionalmente, a maioria dos estudos sobre modularidade em produto abordam a funcionalidade e a redução de custos como enfoque principal, não considerando ou considerando em menor proporção a variável ambiental. Além disso, Arnheiter e Harren (2005) afirmam que embora existam diversos artigos e publicações sobre modularidade, muitas dessas são estudos de caso ou atualizações de revistas comerciais. Uma parcela menor dessas publicações, portanto, são de abordagem teórico-conceitual.

Assim, partindo desse contexto, este trabalho tem como objetivo fazer uma análise exploratória preliminar da literatura acerca das contribuições geradas pela modularidade em produto aos princípios do ecodesign, buscando, dessa forma, preencher a lacuna supracitada nas pesquisas sobre modularidade. Especificamente busca-se ainda investigar os benefícios da modularidade no desenvolvimento de produtos; os princípios e características do ecodesign e inferir, por meio de análise hipotético-dedutiva, os benefícios gerados pela estratégia modular na perspectiva ambiental.

\section{REFERENCIAL TEÓRICO}

Um sistema modular é composto de unidades (ou módulos) que são projetados independentemente, mas que funcionam como um sistema integrado (BALDWIN; CLARK, 1997). A modularidade pode ainda ser considerada uma estratégia para organizar produtos e processos complexos de forma eficiente (BALDWIN; CLARK, 2000; CARIDI et al., 2012).

Em uma perspectiva técnica, a modularidade consiste na composição de produtos complexos em subsistemas mais simples (PANDREMENOS et al., 2009). Esses subsistemas constituem unidades funcionais completas, as quais podem ser projetadas e fabricadas de forma independente (isto é, simultaneamente), o que 
permite o projeto de diferentes produtos (por exemplo diferentes modelos de veículos) por meio da combinação desses subsistemas (BALDWIN; CLARK, 1997; PERSSON, 2006). Ainda segundo os autores citados, esta ação deve permitir o funcionamento integrado do produto, proporcionando uma fácil desmontagem e simples remontagem de produto. Desse modo, duas ideias estão presentes: a de interdependência entre os subsistemas e componentes do próprio módulo e independência (autonomia) entre os módulos (BALDWIN; CLARK, 2000; CAUCHICK MIGUEL, 2004).

Baldwin e Clark (2000) e Morris e Donnelly (2006) distinguem dois tipos de modularidade: no produto e na produção. Há também uma terceira categoria, que é a modularidade de uso (BALDWIN; CLARK, 2000). A pesquisa em modularidade tem se expandido desde a modularidade em produto até a explanação sobre seus efeitos organizacionais, redes de negócios e em setores industriais quanto à gestão do conhecimento e inovação (LANGLOIS, 2002). Em função do foco do presente trabalho, é detalhado neste texto a primeira abordagem, a de modularidade em produto.

A modularidade em produto é um dos fatores mais importantes para determinar a configuração da arquitetura do produto (MIKKOLA; GASSMANN, 2003). Os mesmos autores citam um exemplo: produtos com alto nível de modularidade permitem altas variantes de produto, misturando e combinando os módulos do produto, ao passo que produtos com baixa modularidade permitem a otimização de componentes para um produto em particular. Além disso, a modularidade em produto tem sido visada como uma maneira de acelerar o desenvolvimento de novos produtos e reduzir os custos no processo de desenvolvimento (JACOBS et al., 2011).

$\mathrm{Na}$ modularidade em produto, Morris et al. (2004) definem que a mesma é focada nas fronteiras entre os subsistemas de componentes integrados, dentro de características de projeto e tarefas, as quais devem ser interdependentes entre os módulos (CAUCHICK MIGUEL, 2004). Cauchick Miguel et al. (2009) adicionam que esse tipo de modularidade visa a redução de tempo de fabricação, realizando diferentes atividades simultaneamente e proporciona a concepção de módulos por fornecedores cada vez mais especializados em determinada categoria, o que, 
teoricamente, contribui para melhorias tecnológicas. Além disso, a estratégia modular em produtos, segundo Ando (2004), pode ser implantada em diversos setores da economia, tais como: eletroeletrônicos, telecomunicações, aeronáutica, indústria naval, etc. $\mathrm{Na}$ indústria automobilística, tem-se percebido uma significativa quantia de estudos da modularidade, tanto no Brasil quanto no mundo (CAUCHICK MIGUEL et al., 2009).

As aplicações da modularidade em produto são relatadas na literatura, havendo, inclusive, publicações relacionadas com práticas e estratégias ambientais (foco do presente trabalho). Por exemplo, Nunes e Bennett (2008), em uma pesquisa teórico-conceitual, identificaram contribuições da modularidade para as "operações verdes", tanto na abordagem de produto como outras tais como condomínios modulares e modularidade em cadeia de suprimentos. Christensen (2011), por outro lado, propõe a substituição dos módulos de transmissão automotivos vigentes por módulos que utilizem energias limpas e renováveis (como, por exemplo: elétrica, células combustíveis, dentre outras) e, para isso, menciona quatro veículos os quais utilizam essa concepção. Huang et al. (2012) estruturaram uma nova abordagem metodológica para a modularização e desmontagem de produto considerando os princípios do 3R (Reduzir, Reutilizar e Reciclar), visando melhor disposição final dos produtos após seu consumo.

\subsection{Ecodesign}

A Eco-eficiência se trata de uma filosofia de gestão que visa encorajar o mundo empresarial a procurar melhorias ambientais que, simultaneamente, produzam benefícios econômicos às organizações, por meio de soluções que agreguem mais valor, utilizando menos energia e materiais e, consequentemente, reduzindo o impacto ambiental (BCSD, 2012). O ecodesign é um dos subconjuntos da eco-eficiência (BARBOSA, 2002). Ainda de acordo com o mesmo autor, trata-se de um método para projetos que engloba as variáveis ambientais como parâmetros básicos de projeto para o desenvolvimento de produtos. Ramani et al. (2010) complementam que a metodologia consiste em aplicar critérios ambientais no desenvolvimento e projeto de um produto.

Revista Produção Online, Florianópolis, SC, v.14, n. 2, p. 560-592, abr./jun. 2014. 
termo ecodesign possui diversos sinônimos encontrados na literatura: DFE ou Design for Environment (GARCÍA et al., 2012), Design Sustentável, Design Verde ou Design Limpo (ALMEIDA et al., 2010). No entanto, não necessariamente estes termos podem ser considerados como sinônimos. Karlsson e Luttropp (2006) mencionam que o Ecodesign incorpora as atividades relacionadas à sustentabilidade no cenário global de negócios. "Eco" em Ecodesign pode estar relacionado tanto à economia (refletindo uma orientação empresarial) como à Ecologia, refletindo a importância dos aspectos ambientais (KARLSSON; LUTTROPP, 2006), conforme ilustra a Figura 1.

Figura 1 - Origem do termo Ecodesign

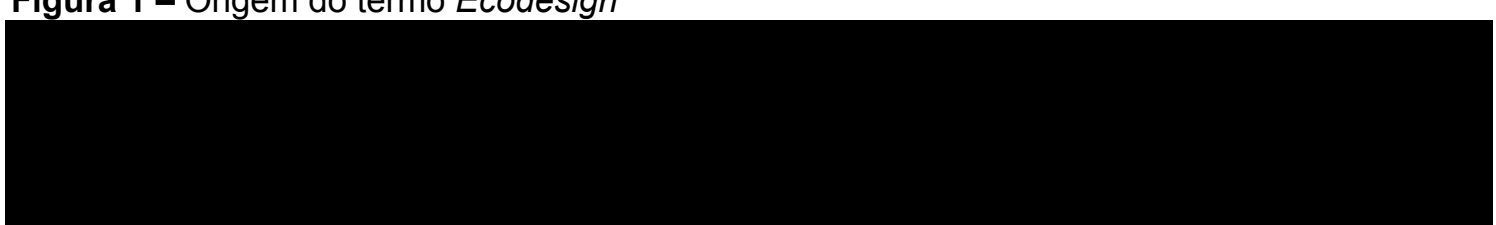

Fonte: adaptado de Karlsson e Luttropp (2006)

Para fins de analisar os benefícios da modularidade em produto, os princípios do ecodesign considerados foram os adaptados de Fiksel (1996), Venzke (2002) e Borchardt et al. (2009), apresentados na Tabela 1.

Tabela 1 - Princípios do Ecodesign e suas respectivas práticas Princípios do
Ecodesign materiais com baixo impacto ambiental

\section{Visar a simplicidade e/ou modularidade do produto}

Práticas e conceitos relacionados (código da prática)

1.1. Reduzir o consumo dos materiais ao longo da vida do produto (produção e utilização).

1.2. Usar matéria-prima mais próximo possível de sua composição natural.

1.3. Evitar a mistura de materiais não compatíveis, o que impede a separação de componentes para reciclagem.

1.4. Usar materiais que geram menos poluição no processo de produção, no uso do produto e posterior reciclagem ou descarte.

1.5. Usar materiais não tóxicos em todas as fases do ciclo de vida do produto (produção, utilização e reciclagem ou descarte).

1.6. Usar materiais reciclados ou materiais que requerem menos energia para produzir.

1.7. Usar materiais que permitem o reuso de componentes.

2.1. Produtos mais simples geralmente envolvem menores custos de produção, menor uso de materiais e permitem uma maior facilidade de montagem e desmontagem.

2.2. Criar produtos cujas peças possam ser substituídas em caso de defeito ou falha, sem a necessidade de substituir o produto em sua totalidade. (continua)

Código da prática

EcoDesign 1.1

EcoDesign 1.2

EcoDesign 1.3

EcoDesign 1.4

EcoDesign 1.5

EcoDesign 1.6

EcoDesign

1.7

EcoDesign

2.1

EcoDesign

2.2

Revista Produção Online, Florianópolis, SC, v.14, n. 2, p. 560-592, abr./jun. 2014. 
Tabela 2 - Princípios do Ecodesign e suas respectivas práticas

Princípios do
Ecodesign $\quad$ Práticas e conceitos relacionados (código da prática)

(conclusão)

Ecodesign

2.3. Fazer componentes de fácil acesso.

Código

da prática

EcoDesign

2.3

3. Incineração

3.1. Ainda que a incineração seja a opção menos preferível de recuperar o valor dos resíduos, é uma opção mais recomendada que a disposição em aterro, especialmente onde há restrição de espaço.

invés de dispô-

los em aterro

3.2. Assegurar que os resíduos incinerados atendam a todos os limites aplicáveis para substâncias perigosas.

4.1. $\mathrm{Na}$ produção: usar equipamentos que sejam eficientes energeticamente e adequadamente dimensionados; usar recursos para economia de energia como a luz natural, por exemplo.

4.2. Na distribuição do produto: considerar toda a energia consumida

4. Reduzir a demanda de energia

\section{Usar fontes de energia renováveis}

\section{Desenvolver produtos multifuncionais}

\section{Desenvolver produtos com maior} durabilidade

8. Recuperar a embalagem do produto

\section{Evitar o uso de substâncias perigosas}

10. Prevenir acidentes na cadeia de distribuição do produto, desde a compra de matériasprimas até a entrega ao consumidor final.

4.3. No uso do produto: desenvolver produtos que incorporem mecanismos para reduzir o consumo de energia, como por exemplo motores mais eficientes, motores que se desligam automaticamente quando não estão em uso, bem como os dispositivos que regulam a potência do motor conforme a demanda.

5.1. Usar fontes renováveis de energia, como por exemplo as energias solar, eólica e hidrelétrica, no lugar de combustíveis fósseis.

6.1. Criar produtos que atendam mais de uma necessidade diferente (isso muitas vezes pode ser realizado utilizando a mesma quantidade de energia e materiais necessários para produtos convencionais de função singular).

6.2. Multifuncionalidade pode ser dividida em dois tipos: (1) a funcionalidade em paralelo, onde o mesmo produto é utilizado simultaneamente para mais do que um propósito, e (2) a funcionalidade sequencial, onde o produto tem uma função primária e, depois, realiza a função secundária.

7.1. Prolongar a vida útil de um produto contribui consideravelmente para a eco-eficiência, uma vez que a durabilidade reduz a necessidade de substituição do produto.

8.1. Gerar um mecanismo que permita a recuperação da embalagem para reutilização ou reciclagem.

8.2. Produtos com opção de recarga são um exemplo de reutilização de embalagens.

9.1. Eliminar do processo produtivo todas as substâncias que podem prejudicar o ambiente ou a saúde dos colaboradores da produção, os consumidores e os funcionários que desmontam o produto para reciclagem.

9.2. Usar componentes à base de água (em particular solventes, adesivos e tintas) como substitutos para os materiais que são à base de hidrocarbonetos.

10.1. Empregar abordagem de prevenção de acidentes na concepção de novos produtos e atualização dos já existentes.

Fonte: adaptado de Fiksel (1996), Venzke (2002) e Borchardt et al. (2009)
EcoDesign

3.1

EcoDesign

EcoDesign

4.1

EcoDesign

4.2

EcoDesign

4.3

EcoDesign

5.1

EcoDesign

6.1

EcoDesign

6.2

EcoDesign

7.1

EcoDesign

8.1

EcoDesign

8.2

EcoDesign

9.1

EcoDesign

9.2

EcoDesign 10.1

\section{MÉTODO DE PESQUISA}

O presente trabalho pode ser classificado como teórico-conceitual (conforme

BERTO; NAKANO, 2000), uma vez que considera uma revisão de literatura e a

Revista Produção Online, Florianópolis, SC, v.14, n. 2, p. 560-592, abr./jun. 2014. 
busca de análise da teoria vigente nos temas abordado. Para a realização do estudo, foram considerados dois campos do conhecimento: modularidade (enfoque em produto) e ecodesign, seus princípios e aplicações. Assim, buscou-se publicação qualificada relevante de artigos publicados, preferencialmente, em periódicos classificados segundo o Qualis-CAPES (Coordenação de Aperfeiçoamento de Pessoal de Ensino Superior).

A busca bibliográfica consistiu na recuperação de artigos científicos acerca dos referidos temas (modularidade em produto e ecodesign). A busca se deu por meio das expressões-chave relacionadas com os temas: "modularity", "modularization" (e variantes, por exemplo: "modularisation"), "modular product", "modular design", "green design", "clean design", "Design for Environment", "DFE", "sustainable design", "eco-innovation", "Green product development", "sustainable product development", "environmentally friendly products", "green product", "green lifecycle", "green product design", "design for recyclability", "design for sustainability" e "design for rebirth". É importante ressaltar que para uma melhor filtragem, os artigos que possuíam as expressões-chave no título, resumo e/ou palavras-chave foram considerados para análise.

Foram considerados os artigos que relacionam os conceitos de modularidade e suas aplicações em produto (projeto) com as abordagens do ecodesign para, em seguida, apresentar os benefícios e recomendações dessa combinação. Para isso, fez-se uma busca estruturada, com suporte do software EndNote $X 5^{\circledR}$ para registro, nas bases de dados Scopus, ISI Web of Knowledge, Science Direct e Scielo. Inicialmente, considerou-se o período de 10 anos (2003-2012). Entretanto, essa busca resultou em uma baixa quantidade de artigos para análise. Assim, fez-se uma posterior busca não estruturada para aumentar o número de trabalhos analisados, considerando o mesmo período. Estes detalhes são apresentados na seção 4.1 dos resultados.

Como descrito, no primeiro momento da busca estruturada, encontrou-se um número limitado de artigos, sendo que nem todos estavam disponíveis de forma aberta (acesso restrito). Posteriormente, realizou-se uma verificação nas referências dos estudos encontrados inicialmente na busca estruturada e, posteriormente, nas 
bases de dados citadas. Dessa forma, foi possível encontrar uma quantidade maior de publicações sobre a modularidade em produto aplicada ao ecodesign.

A partir desses dados, utilizou-se como principal técnica de análise o método hipotético-dedutivo (similarmente ao adotado por NUNES; BENNETT, 2008), inferindo as principais contribuições da modularidade (conforme análise dos autores do presente artigo) em cada um dos princípios do ecodesign, obtendo-se, dessa forma, descobertas e posicionamentos a partir da literatura no tema. A escolha dessa técnica se justifica pelo fato da necessidade de pesquisas teóricas mais aprofundadas sobre o tema, como relatado na seção 2 deste trabalho. A Figura 2 sintetiza a estrutura metodológica descrita e a Tabela 1 (referencial teórico) apresentou os itens avaliados na análise de conteúdo com os respectivos códigos de cada prática (seção 4.2).

Figura 2 - Metodologia da pesquisa

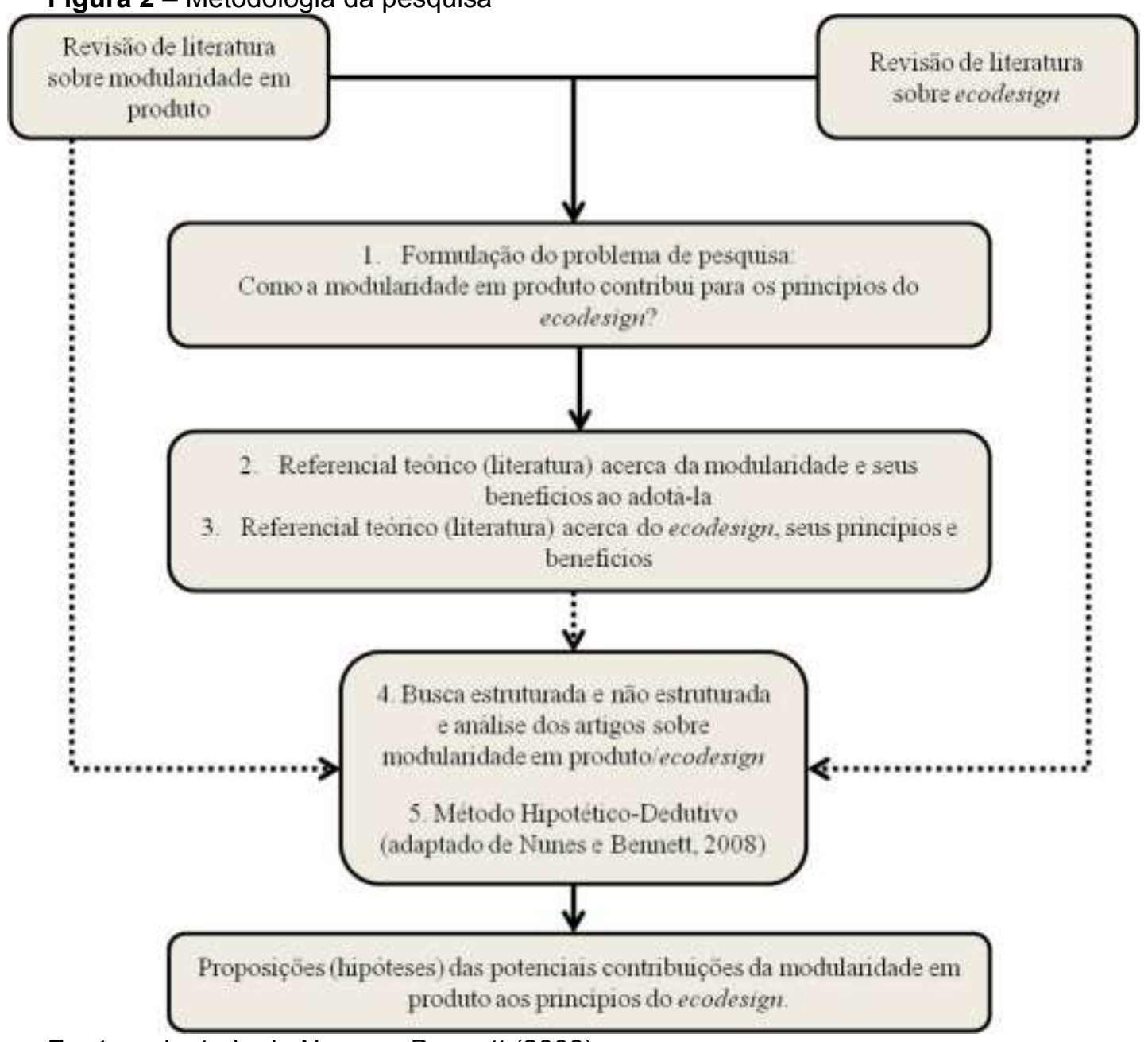

Fonte: adaptado de Nunes e Bennett (2008).

Revista Produção Online, Florianópolis, SC, v.14, n. 2, p. 560-592, abr./jun. 2014. 


\section{RESULTADOS E DISCUSSÃO}

Os resultados são subdivididos em uma análise descritiva das publicações identificadas para, em seguida, realizar uma análise de conteúdo das publicações resultantes.

\subsection{Levantamento e análise das publicações encontradas}

Antes de se realizar as inferências quanto às contribuições da modularidade em produto ao ecodesign, foi conduzida uma análise bibliométrica descritiva dos artigos selecionados. Desse modo, foram encontradas publicações nos periódicos mostrados na Tabela 2.

Tabela 3 - Periódicos e quantidade de publicações

\begin{tabular}{c|c}
\hline Pournal of Cleaner Production & Quantidade \\
\hline Robotics and Computer-Integrated Manufacturing & 3 \\
Expert Systems with Applications & 2 \\
Chinese Journal of Mechanical Engineering & 2 \\
Computers \& Industrial Engineering & 2 \\
Computers in Industry & 2 \\
CIRP Annals - Manufacturing Technology, International Journal of Advanced & 2 \\
Manufacturing Technology, International Journal of Environmental & 1 \\
Technology and Management, International Journal of Production Research, & \\
International Journal of Sustainable Manufacturing, Computer, Journal of & \\
Environmental Management, Applied Energy, IEEE Transactions on & \\
Electronics Packaging Manufacturing, Brazilian Journal of Operations \& & \\
Production Management, Journal of Purchasing \& Supply Management, & \\
Gestão \& Produção, International Journal of Operations \& Production & \\
Management, Journal of Mechanical Design, Renewable \& Sustainable & \\
Energy Reviews, Journal of Dalian University of Technology e Journal of & \\
Shanghai Jiaotong University (Science) & \\
\hline
\end{tabular}

Fonte: dados da pesquisa.

Como pode ser visto na Tabela 2, a busca estruturada retornou um total de 22 artigos relacionando os dois temas deste trabalho (modularidade em produto e ecodesign). Um desses trabalhos foi desconsiderado por não ter relação evidente das duas abordagens, sendo totalizado, assim, 21 artigos, uma quantidade 
considerada pequena para análise. Devido a isso, posteriormente, procedeu-se um segundo levantamento não estruturado de publicações acerca do assunto, com o objetivo de avaliar uma maior quantidade de trabalhos e proporcionar maior fundamentação teórica para as inferências. Desse modo, identificou-se mais 9 artigos por meio de uma busca exploratória, que se deu nas mesmas bases de dados já mencionadas nos procedimentos metodológicos (seção 3) deste trabalho, totalizando, assim, para fins de análise de conteúdo, 30 artigos abordando o tema modularidade em produto e ecodesign.

Depois de uma primeira verificação das publicações identificadas, algumas características são destacadas:

- A maioria dos artigos (63\%) são de caráter teórico, ou seja, propõe métodos e modelos conceituais de desenvolvimento de produtos, considerando a modularidade em produto alinhada a aspectos do ecodesign e design sustentável, seguidas de estudo ou análise de casos para a avaliação dos modelos propostos. Uma estratificação é mostrada na Figura 3;

- As publicações sobre os dois temas estão disponíveis em periódicos diversos (30 publicações disponíveis em 23 periódicos diferentes, como visto na Tabela 2), não havendo, assim, uma base de referência de destaque sobre o assunto. O periódico com mais ocorrências foi o Journal of Cleaner Production, com três publicações, seguido de outros cinco periódicos com duas publicações cada: Expert Systems with Applications, Robotics and Computer-Integrated Manufacturing, Computer \& Industrial Engineering, Computers in Industry e Chinese Journal of Mechanical Engineering. Esses periódicos abrangem $43 \%$ do total das publicações;

- No levantamento de palavras-chave, destacaram-se: "product design" (8), "modularity" (8), "modular design" (6) e "lifecycle" (4). A palavra-chave "Ecodesign" obteve três ocorrências;

- Em relação ao aspecto temporal das publicações, notou-se um aumento de pesquisas recentes sobre a modularidade no ecodesign. Das 30 publicações encontradas, $80 \%$ foram realizadas a partir de 2008 ;

- Notou-se que uma parcela maior dos estudos tem como enfoque a modularidade contribuindo no final do ciclo de vida dos produtos, por meio da melhora nos 
fatores de reaproveitamento de componentes e reciclabilidade dos módulos na disposição final dos produtos;

- A maior parte dos trabalhos tem aplicação geral (37\%), ou seja, não foca em um setor específico. Os setores da economia mais abordados são a indústria automotiva (20\%), eletrodomésticos (13\%) e eletroeletrônicos (10\%) - ilustração dos resultados se encontra na Figura 4. A evidência sobre o setor automotivo pode ser corroborada pela literatura, pois este segmento vem crescentemente utilizando a estratégia. Nunes e Bennett (2008) ainda reforçam que o setor automotivo é criticado constantemente devido à sua alta carga de impactos ambientais negativos, bem como seu comportamento conservador e reativo que produz poucas inovações radicais. Orsato e Wells (2007) corroboram, citando que um dos principais desafios da indústria automobilística é a cobrança por minimizar (ou até mesmo eliminar) as emissões nocivas dos veículos, buscar o uso racional de recursos naturais e também lidar com impactos como os congestionamentos e os acidentes com fatalidades. Ademais, o setor de eletrônicos foi um dos pioneiros na estratégia de modularidade, como relatado em Baldwin e Clark (1997). Quanto à indústria de linha branca, a possível motivação para a utilização da modularidade é a facilidade de desmontagem e reaproveitamento de recursos dos produtos (KUO, 2006).

Figura 3 - Tipos de pesquisa predominantes dos estudos

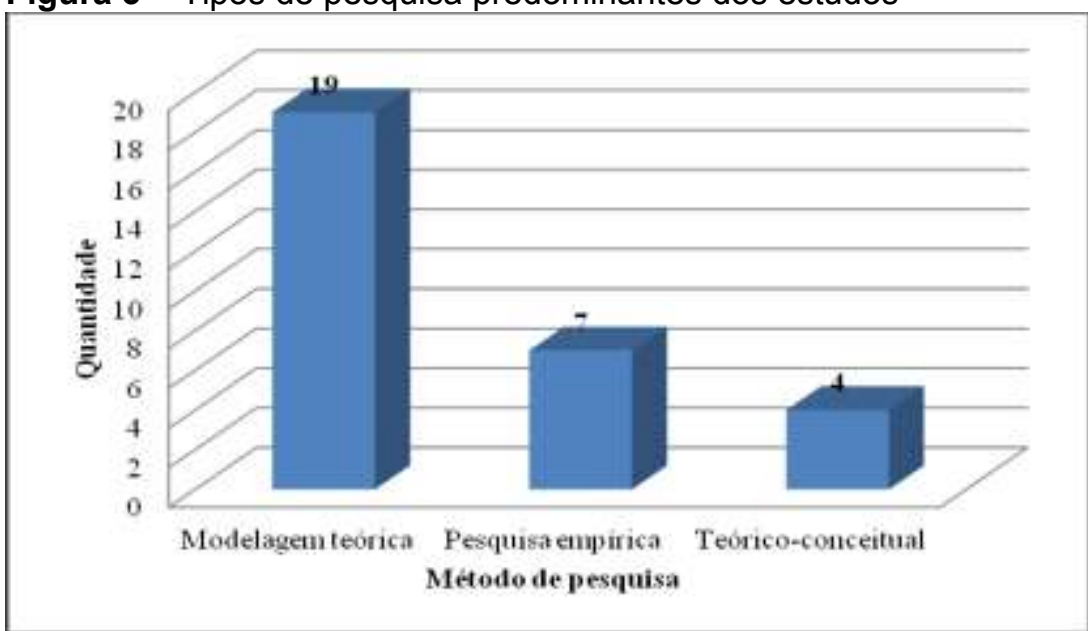

Fonte: Autores, dados da pesquisa

Revista Produção Online, Florianópolis, SC, v.14, n. 2, p. 560-592, abr./jun. 2014. 
Figura 4 - Setores industriais mais estudados.

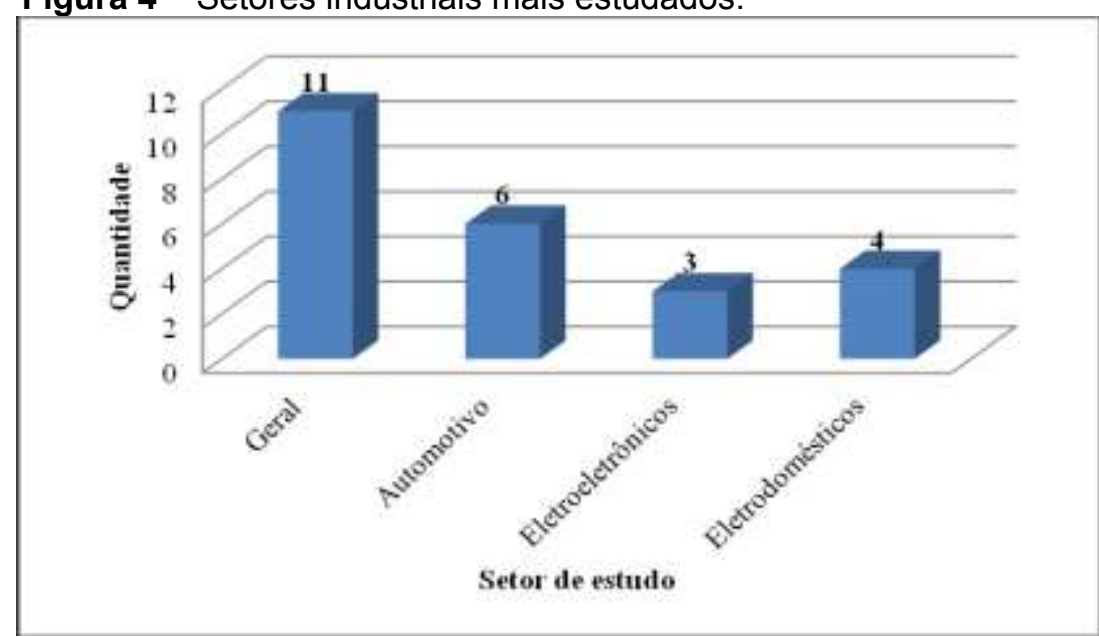

Fonte: Autores, dados da pesquisa.

Observa-se assim a predominância de modelos teóricos amplos, que podem ser aplicados em diversos setores da economia, além da adoção de modelagem teórica como principal método de pesquisa. Ressalta-se ainda que esses estudos têm sido desenvolvidos principalmente nos últimos anos, emergindo como um tema de interesse recente nas publicações sobre modularidade. Para uma melhor compreensão dos trabalhos e identificação de oportunidades futuras, posteriormente foi feita a análise de conteúdo dos artigos, visando extrair as contribuições da modularidade em produto aos princípios do ecodesign, descritas na próxima subseção.

\subsection{Contribuições da modularidade em produto aos princípios do ecodesign}

Após a realização da busca na literatura sobre a modularidade e ecodesign, procedeu-se à identificação das possíveis contribuições da modularidade em produto à estratégia de projeto para o meio ambiente. Como anteriormente destacado, os princípios do ecodesign considerados foram os adaptados de Fiksel (1996), Venzke (2002) e Borchardt et al. (2009). De modo a organizar os resultados obtidos, as contribuições da modularidade são apresentadas a seguir, a partir dos princípios do ecodesign (Tabela 1), encontrados a partir da análise dos artigos apresentados na seção 4.1 .

Revista Produção Online, Florianópolis, SC, v.14, n. 2, p. 560-592, abr./jun. 2014. 


\subsubsection{Escolha de materiais com baixo impacto ambiental}

\subsubsection{Redução do consumo dos materiais (EcoDesign 1.1 na Tabela 1)}

Nesta prática, pode-se associar a modularidade no sentido de a estratégia possibilitar a redução de consumo de materiais por meio da customização dos módulos, o que gera economia de escala (LAU et al., 2009; HUANG et al., 2012). Huang et al. (2012) mencionam também a facilidade de manutenção, reparos, descarte e reuso utilizando-se da estratégia modular. Essa economia não ocorre somente em recursos humanos e financeiros, mas também de matéria-prima, energia e descarte ou reutilização, uma vez que é minimizada a necessidade de produção de novas peças, componentes e tratamento final de resíduos. Este fato contribui, assim, para um menor impacto ambiental do produto ao longo do seu ciclo de vida.

4.2.1.2 Utilizar matéria-prima próxima da composição natural (EcoDesign 1.2 na Tabela 1)

Aqui podem ser inseridas contribuições da modularidade em produto pelo fato de a dissociação de componentes permitir uma seleção mais flexível de materiais. Desse modo, as oportunidades da utilização de matéria-prima mais próxima das composições naturais são potencializadas, conforme a necessidade e/ou prioridade na elaboração dos projetos.

4.2.1.3 Utilizar matéria-prima próxima da composição natural (EcoDesign 1.3 na Tabela 1)

A possibilidade de divisão de um produto complexo em subcomponentes mais simples contribui potencialmente para eliminar a mistura de materiais em determinada estrutura de um produto. Huang et al. (2012) mencionam a facilidade de reuso e reciclagem com a adoção da estratégia modular em produtos. Entretanto, para que essas ações possam ser efetuadas na prática, é necessário, 
adicionalmente, o cuidado para que os produtos não compilem materiais incompatíveis, pois caso contrário, o reaproveitamento e destinação correta, ao fim do ciclo de vida, serão comprometidos.

\subsubsection{Usar materiais menos poluentes (EcoDesign 1.4 na Tabela 1)}

De modo geral, alguns exemplos podem ser encontrados na literatura, em especial na indústria automotiva, grande geradora de impactos ambientais (NUNES; BENNETT, 2008; CHRISTENSEN, 2011).

Nunes e Bennett (2008) citam o projeto Ridek (veículo modular) como um conceito de modularidade que considera o uso do produto como uma inovação radical, onde a flexibilidade do conceito faz com que o veículo se adapte tanto nas necessidades urbanas como em rodovias, trazendo contribuições quanto a minimização da poluição do ar, além de ser produzido em pequena escala, o que também gera menos resíduos e emissões. Além disso, há também o modelo híbrido produzido pela Toyota $^{\circledR}$, o Prius (TOYOTA, 2012), que combina motores elétricos e de combustão em seu sistema, para determinar o funcionamento mais eficiente do veículo. Tal combinação, além de proporcionar melhor desempenho ao automóvel, minimiza as emissões atmosféricas durante o uso do produto pelo consumidor.

Christensen (2011) relata também que a modularidade de sistemas de transmissão está fortemente relacionada com a fonte de energia elétrica, em especial as baterias. Desse modo, o autor citado afirma que a futura viabilidade de soluções alternativas de transmissão tem 0 avanço nesse módulo, pelos fornecedores, como um fator crítico de sucesso. Soluções nesta direção carregam potencial redução de poluição.

\subsubsection{Usar materiais atóxicos (EcoDesign 1.5 na Tabela 1)}

A não utilização de materiais tóxicos é uma condição desejável independentemente da arquitetura a ser utilizada no produto (integral ou modular). Entretanto, sabe-se que conforme o tipo de produto, bem como função que realizará e mercado de atuação, essa condição pode não ser obtida em sua totalidade. Com a 
adoção de projeto de produto modular, facilita-se a disposição e a localização no uso desses materiais na estrutura do mesmo, o que permite a destinação correta do material sem elevados impactos ambientais negativos, após sua utilização, bem como não compromete as partes reaproveitáveis e possíveis de um melhor direcionamento pelo fato de estarem inseridas em outros módulos do produto.

4.2.1.6 Usar materiais reciclados ou que demandam pouca energia (EcoDesign 1.6 na Tabela 1)

O desenvolvimento de produtos modulares contribui na economia de recursos para viabilização dos mesmos. Dentro dessa perspectiva, insere-se o uso de materiais que requeiram menos energia e/ou com alto nível de reciclabilidade. Essa prática pode ser benéfica principalmente, por exemplo, na indústria de eletrônicos, que se destaca no uso da modularidade em produtos. Com a utilização de materiais reciclados, tem-se maior possibilidade de, ao final do uso dos produtos, encaminhálos para reaproveitamento no processo produtivo, por meio da reciclagem ou processos que utilizem menos recursos para transformá-los novamente em matériaprima para a produção de novos equipamentos.

\subsubsection{Usar materiais que permitem reuso (EcoDesign 1.7 na Tabela 1)}

Em relação ao reuso de componentes, Arnheiter e Harren (2005) afirmam que visando produtos ambientalmente aceitáveis, os projetistas devem ter atenção cuidadosa com os módulos de vida útil limitada. Devido a curta duração e alta rotatividade desses componentes, a reciclagem e o uso responsável dos recursos naturais devem ser considerados ao longo do desenvolvimento de produtos (ARNHEITER; HARREN, 2005). Os mesmos autores citam um exemplo: empresas que vendem impressoras e recolhem os cartuchos e toners usados para retornar aos respectivos fabricantes. Os mesmos podem ser recarregados e reutilizados, o que diminui o descarte e consequente prejuízo ambiental.

Além disso, o reuso pode economizar recursos financeiros, pois um pedido por um novo produto não será necessário, o que economiza dinheiro e tempo (GO et 
al., 2012). Ressalta-se que o reuso é importante para a sustentabilidade devido aos seguintes aspectos: reduz a necessidade de disposição em aterro e/ou incineração, evita a poluição causada pela fabricação de produtos a partir de matéria-prima nova, economiza energia, reduz as emissões de gases estufa que contribuem para as mudanças climáticas globais, conserva recursos naturais como madeira, água e minerais, protege e expande trabalhos de fabricação e aumenta a competitividade dos fabricantes e, por fim, ajuda a manter o meio ambiente às futuras gerações (SUSTAINABLE JERSEY, 2010; GO et al., 2012).

\subsubsection{Visar a simplicidade e/ou modularidade do produto}

O próprio conceito de modularidade está fortemente alinhado a este princípio, uma vez que o objetivo dessa estratégia é subdividir um produto complexo em módulos simplificados, que facilitem o gerenciamento de sistemas complexos, a montagem e desmontagem dos produtos (BALDWIN; CLARK, 1997; BALDWIN; CLARK, 2004; PANDREMENOS et al., 2009). A modularidade no compartilhamento de componentes, por exemplo, é utilizada para potencializar economias de escopo, uma vez que o mesmo componente (módulo) é utilizado em diversos produtos (MACHADO; MORAES, 2008).

4.2.2.1 Desenvolver produtos mais simples para economia de recursos (EcoDesign 2.1 na Tabela 1)

Baldwin e Clark (2000) e Arnheiter e Harren (2005) mencionam os principais benefícios da modularidade: simplificação do processo de projeto, aumento da produtividade no projeto de produto, possibilidade de redução de custos devido ao uso de módulos comuns em diferentes produtos e auxílio na customização em massa. Benefícios os quais têm forte relação com o ecodesign, no que se refere, principalmente, ao menor uso de materiais para a execução do projeto de produto, contribuindo para um consumo racional de recursos no desenvolvimento de produtos.

Revista Produção Online, Florianópolis, SC, v.14, n. 2, p. 560-592, abr./jun. 2014. 
A facilidade de montagem e desmontagem também é importante na reutilização e descarte dos componentes, tendo relação, adicionalmente, com práticas do primeiro princípio do ecodesign (itens 1.1 e 1.7 - Tabela 1). Outro exemplo de benefício neste princípio é citado em Veloso e Fixson (2001), onde os autores destacam a redução de complexidade do produto no caso de montadoras automotivas. Assim, dentro do conceito básico de modularidade (subdivisão de produto complexo em componentes mais simples), há a oportunidade de reduzir os custos de produção e consumo de matéria-prima e energia.

4.2.2.2 Desenvolver produtos com componentes substituíveis (EcoDesign 2.2 na Tabela 1)

A criação de produtos com peças substituíveis em caso de defeito ou falha contribui para eliminar ou minimizar a substituição total de um produto em caso de problemas e/ou fim de vida útil. Essa é outra prática a qual é alinhada à estratégia modular em produtos. Exemplos disso são relatados por Arnheiter e Harren (2005) quanto às baterias de carro e Murphy (2002), que menciona as pastilhas de freios de um veículo automotivo que podem ser substituídas, sem a necessidade de trocar todo o sistema de freio.

\subsubsection{Desenvolver componentes de fácil acesso (EcoDesign 2.3 na Tabela 1)}

A partir do conceito clássico de modularidade de Baldwin e Clark (1997), que define modularidade como a partição de um produto complexo em subcomponentes mais simples, isso pode ser facilmente observado. A simplificação de produtos complexos por meio da modularidade gera, consequentemente, a utilização e disponibilização de componentes com maior acessibilidade e facilidade de uso.

\subsubsection{Incineração de resíduos ao invés de dispô-los em aterro}

4.2.3.1 Incinerar resíduos onde há restrição de espaço (EcoDesign 3.1 na Tabela 1) 
Não foram encontradas contribuições diretas da modularidade a essa prática (escolha da incineração ao invés de disposição em aterro). Entretanto, a separação do produto em diversos módulos (conforme o nível de modularidade) pode facilitar a dissociação de componentes para posterior destinação para aterros (quando não houver possibilidade de recuperação) ou, no caso em questão, para incineração. Dentro do contexto desta prática, torna-se relevante mencionar que é preferível minimizar, inicialmente, o consumo de recursos no desenvolvimento e utilização do produto, o reaproveitamento de seus componentes ou a reciclagem dos mesmos, evitando-se ao máximo chegar ao estágio de incineração e aterro, uma vez que são práticas que também consomem recursos e também podem gerar impactos ambientais negativos.

4.2.3.2 Estar em conformidade com os limites para o uso de substâncias perigosas (EcoDesign 3.2 na Tabela 1)

Assim como na prática anterior (4.2.3.1.), não foram encontradas na literatura contribuições diretas da modularidade em assegurar o atendimento aos requisitos para resíduos incinerados. No entanto, produtos modulares têm mais facilidade de incineração (caso necessário), pois sua arquitetura permite uma melhor separação dos componentes que poderão ser reaproveitados e os que serão descartados.

\subsubsection{Reduzir a demanda de energia}

4.2.4.1 Produção: utilizar equipamentos mais eficientes e melhor dimensionados (EcoDesign 4.1 na Tabela 1 )

Considerando apenas a perspectiva de modularidade em produto e seu conceito, esta prática do ecodesign não tem relação direta. Entretanto, partindo-se para a modularidade na produção (estruturação do processo produtivo em módulos), é possível notar que a produção modular pode ser benéfica no sentido de utilizar quantidade racional de energia para a fabricação dos produtos. Lorenzi e Lello (2001) complementam dentro do ponto de vista de redução de tempo e maior 
agilidade na modularidade em processo para atender às demandas. Esta rapidez gera, consequentemente, menor consumo energético bem como evita o excesso de gasto dos mesmos. Por outro lado, há trabalhos que, por meio da modularidade em produto, trazem contribuições nos processos que os viabilizam. Exemplo disso é o estudo feito por Fernandez e Kekäle (2005), que analisa os impactos da modularidade em produto sob a perspectiva da logística reversa. Além disso, uma prática que contribui de forma relevante para esse conceito, embora não esteja no escopo da modularidade em produto, são os condomínios (ou consórcios) modulares (FIRMO; LIMA, 2005; SALERNO et al., 2009; SALERNO et al., 2008), ou seja, empresas que compartilham uma mesma planta industrial para uso de recursos. Em adição aos benefícios de redução de custos com matéria-prima e energia há, consequentemente, a redução de consumo de recursos não renováveis, como a própria energia elétrica, vapor, água, etc.

4.2.4.2 Distribuição do produto: considerar energia consumida na cadeia de distribuição (EcoDesign 4.2 na Tabela 1)

O consumo de energia ao longo da cadeia de suprimentos pode ser reduzido com a adoção da modularidade. Esta situação pode ser inferida com base no estudo de Lau et al. (2009). Os autores afirmam que um dos benefícios da modularidade é a dissociação entre as tarefas de desenvolvimento e a possibilidade de desenvolver componentes de forma concorrente (ou simultânea) para encurtar o tempo de desenvolvimento do produto, benefício também é mencionado por Carnevalli et al. (2011). Ainda, Lorenzi e Lello (2001) afirmam que a modularidade impacta positivamente no tempo de desenvolvimento. Ou seja, o desenvolvimento de módulos possibilita o uso de conceitos da engenharia simultânea, contribuindo para, além da redução de custos, a diminuição no consumo de recursos energéticos ao longo da distribuição e concepção do produto. Exemplo dessa vantagem é o estudo realizado por Carvalho et al. (2012), que propuseram a redução de consumo de combustível em veículos automotivos por meio da arquitetura modular de produto. Por meio do modelo matemático elaborado pelos autores, concluíram que há a possibilidade de redução de consumo de combustível por meio de veículos 
modulares e mais leves, partes (módulos) ajustáveis e customizáveis e mudanças culturais por parte dos consumidores.

4.2.4.3 Uso do produto: desenvolver mecanismos para reduzir o consumo de energia (EcoDesign 4.3 na Tabela 1)

Dentro da perspectiva de Carvalho et al. (2012), que propuseram um modelo matemático para a elaboração de veículos modulares mais eficientes do ponto de vista energético, percebeu-se que a modularidade na arquitetura de produto tem potencial para, inclusive, reduzir o consumo de combustível dos automóveis por meio de estruturas mais leves. Outro exemplo de mecanismo que reduz o consumo de energia é o veículo Toyota ${ }^{\circledR}$ Prius (TOYOTA, 2012). O referido automóvel possui sistema de relação potência/consumo que opera por meio de um motor elétrico que funciona juntamente com o motor movido a combustível fóssil, gerando uma redução no consumo deste recurso, além de o motor a combustível se desligar quando o veículo está parado. Além disso, há a opção de utilização apenas do motor elétrico a baixas velocidades (de 2 a $50 \mathrm{~km} / \mathrm{h}$ ), o que beneficia a minimização de emissões (TOYOTA, 2012).

\subsubsection{Usar fontes de energia renováveis}

\subsubsection{Usar fontes renováveis de energia (EcoDesign 5.1 na Tabela 1)}

O uso de módulos que consumam energias limpas e renováveis é um tipo de inovação de impacto significativo, em especial no setor automotivo, onde o consumo de combustíveis fósseis é elevado. Como exemplo de potenciais contribuições nesse princípio, pode-se mencionar o estudo desenvolvido por Christensen (2011), onde o autor discute e apresenta possibilidade de eco-inovação nos sistemas de transmissão de automóveis, e também Ally e Pryor (2009), que estudaram a implantação de chassis a células de hidrogênio em ônibus, visando maior eficiência ambiental e energética. Ally e Pryor (2009) pesquisaram as barreiras e necessidades na fabricação em massa de chassis de ônibus usando hidrogênio como combustível, 
por meio de uma arquitetura de produto modular com sistema de transmissão híbrida, e também de estudos para a construção de módulos de armazenamento de energia efetivos, células combustíveis e baterias intercambiáveis, interface padrão entre módulos, entre outras práticas para produtos mais limpos.

Christensen (2011) analisa como a modularidade pode contribuir para a utilização de sistemas alternativos de transmissão em automóveis, e para isso, cita e discute quatro casos dessa possibilidade: GM Hy-wire (células combustível e sistema drive-by-wire), TH!NK (células de hidrogênio, motor elétrico e recarregável), Chevy Volt (sistema de transmissão elétrica e três diferentes combinações de propulsão: apenas bateria elétrica, combinação de motor elétrico/motor a combustão e combinação elétrica/célula combustível) e o Mitsubishi MIEV - motor acoplado às rodas, que permite combinação entre carros movidos a bateria elétrica, híbridos com energia elétrica ou os veículos com a combinação entre motor elétrico e célula de hidrogênio (CHRISTENSEN, 2011). Nesta prática, também se pode considerar o Toyota $^{\circledR}$ Prius (TOYOTA, 2012), já mencionado nas práticas 4.2.1.4 e 4.2.4.3 (utilização de energia elétrica para economia de combustível fóssil).

\subsubsection{Desenvolver produtos multifuncionais}

4.2.6.1 Desenvolver produtos com diversas funcionalidades (EcoDesign 6.1 na Tabela 1)

Por meio da adoção da modularidade, é possível criar produtos que atendam diversas funcionalidades por intermédio da construção de partições (módulos) que tenham a capacidade de, individualmente, desempenhar funções que atendam as necessidades dos consumidores, além da função global do produto como um todo. Dessa forma, economiza-se recursos de energia e materiais para a realização de diversas funções ao invés de se fazer produtos diversos que atendam as mesmas funções. Complementando, a geração desses produtos multifuncionais pode ser viabilizada fazendo com que os módulos (partições) realizem funções individuais, ao passo que o produto, com a união dessas funções, executa a função global para o qual foi projetado.

Revista Produção Online, Florianópolis, SC, v.14, n. 2, p. 560-592, abr./jun. 2014. 
4.2.6.2 Utilizar a multifuncionalidade em paralelo e sequencial (EcoDesign 6.2 na Tabela 1)

Uma das principais vantagens da utilização da estratégia modular em produtos é a possibilidade de se gerar produtos multifuncionais. Isso pode ser concluído a partir do conceito básico (mas fundamental) de modularidade de Baldwin e Clark (2000), onde os autores afirmam que é a partição de um produto complexo em diversos componentes (módulos) separados, mas que funcionam como um todo. Sendo assim, cada módulo realiza, individualmente, uma função específica na arquitetura do produto, e a união desses componentes visa o desenvolvimento pleno da função global do mesmo. Existem diversos exemplos práticos dessas multifuncionalidades no mercado. Computadores são subdivididos em diversos módulos, dentre alguns: placa-mãe, processador, memória e bateria. Cada um desses módulos possui uma função dentro do produto (exemplo: bateria fornece energia para que o computador funcione) e a função de cada um dos componentes converge para a viabilização da utilização do computador por parte do usuário.

No caso da funcionalidade em paralelo, os telefones celulares atuais são exemplos: além de realizar a sua função principal (comunicação de voz), o aparelho, conforme o modelo, pode ser utilizado como agenda de compromissos e anotações, despertador, relógio, acesso à internet e redes sociais, envio de mensagens, dentre outras funcionalidades. Isso significa que o produto pode realizar várias funções ao mesmo tempo, não sendo mais necessário, na prática, a aquisição de diversos produtos para realizar cada uma das funções supracitadas (agenda física e relógio despertador, por exemplo). Nesse caso, a modularidade em produto está mais relacionada aos softwares ao invés das partes físicas do produto.

\subsubsection{Desenvolver produtos com maior durabilidade}

\subsubsection{Prolongar a vida útil dos produtos (EcoDesign 7.1 na Tabela 1)}

O desenvolvimento de produtos com maior durabilidade não é uma ação restrita apenas à adoção da modularidade em produto. Visando maior eco-eficiência, 
há diversas estratégias para se melhorar a durabilidade de produtos. Considerando os conceitos e benefícios da modularidade em produto, não foi possível inferir benefícios diretos da abordagem a esta prática do ecodesign. Entretanto, o que se pode observar é a facilidade para testes de resistência dos módulos, pois em caso de falhas, perde-se menos recursos do que a realização de testes ao final do produto integral.

\subsubsection{Recuperar a embalagem do produto}

4.2.8.1Recuperar embalagens para reuso ou reciclagem (EcoDesign 8.1 na Tabela 1)

A adoção da modularidade no projeto de produto facilita a geração de mecanismos que recuperem, além das partes e componentes modulares, as embalagens dos produtos, pois se consegue a melhor separação dos componentes (nisso se inclui a embalagem), prática que consequentemente facilita a recuperação máxima de todas as partes dos produtos. Ainda, trata-se de uma prática que pode ser observada na indústria de linha branca (FERNANDEZ, KEKÄLE, 2005).

\subsubsection{Desenvolver produtos com opção de recarga (EcoDesign 8.2 na Tabela 1)}

Assim como na prática anterior (4.2.8.1), a modularidade permite o desenvolvimento de componentes, peças ou subsistemas que possam ser recarregados e substituídos, ao invés da troca completa do produto. Nos exemplos relatados em Christensen (2011) isso pode ser evidenciado, uma vez que os módulos de transmissão elétrica podem ser reabastecidos à medida que são utilizados. Essa recarga também pode ser mostrada com o caso do Toyota Prius (TOYOTA, 2012).

\subsubsection{Evitar o uso de substâncias perigosas}

\subsubsection{Eliminar substâncias nocivas (EcoDesign 9.1 na Tabela 1)}


A modularidade tem potencial contribuição neste aspecto no que se refere, principalmente, à reciclagem. Produtos modulares têm maior facilidade de serem direcionados de forma correta após o seu uso pelo consumidor (HUANG et al., 2012). Essa facilidade se deve, também, ao fato de se conhecer, com maior precisão, os elementos que compõem cada módulo do produto. Assim, aqueles com materiais e/ou substâncias mais nocivas podem ser direcionados para uma manipulação pós-uso mais minuciosa, ao passo que os componentes menos perigosos são direcionados para atividades mais simples de reuso/reciclagem.

\subsubsection{Usar componentes à base de água (EcoDesign 9.2 na Tabela 1)}

Os módulos os quais se utilizam de hidrocarbonetos podem ser mais bem investigados para que os mesmos tenham evoluções relacionadas à adoção de materiais e composições menos agressivas ao meio ambiente. Desse modo, o aprofundamento de estudos por módulos pode se tornar mais viável em relação ao estudo do produto como um todo, por meio da decomposição do produto (simplificando a compreensão de sua estrutura) e posterior composição dos subsistemas. Exemplos de módulos que têm potencial para substituir os hidrocarbonetos podem ser encontrados na indústria automobilística, nos casos já mencionados em práticas anteriores do ecodesign (NUNES; BENNETT, 2008; ALLY; PRYOR, 2009; CHRISTENSEN, 2011).

\subsubsection{Prevenir acidentes}

4.2.10.1 Utilizar abordagem de prevenção de acidentes (EcoDesign 10.1 na Tabela 1)

Poucas evidências foram encontradas dos benefícios da modularidade nesse princípio, na literatura. Lau et al. (2009) afirma que a modularidade melhora a confiabilidade do sistema devido ao elevado volume de produção e a curva de experiência adquirida. Partindo-se dessa vantagem, é possível observar que o aumento de confiabilidade e experiência proporciona maior segurança aos produtos, 
bem como seus componentes (módulos), reduzindo, assim, a probabilidade de acidentes durante o desenvolvimento, consumo e destinação final do produto.

Assim, percebe-se uma considerável contribuição da modularidade aos princípios do ecodesign. Em seguida, são relatadas as conclusões referentes ao trabalho, bem como suas limitações e oportunidades para estudos futuros.

\section{CONCLUSÕES}

Este trabalho teve como objetivo principal investigar quais contribuições a estratégia de modularidade em produto proporciona aos conceitos do ecodesign, possibilitando uma perspectiva ambiental da modularidade no desenvolvimento de novos produtos e também redesenho de produtos já existentes. Por meio da análise preliminar da literatura, pôde-se perceber que há um número bastante limitado de estudos acerca do assunto disponíveis.

Inicialmente, notou-se que as publicações relacionando os dois temas estão distribuídas em diversos periódicos, fator que pode dificultar a pesquisa no assunto, mas que demonstra uma ampla gama de interesse em termos de fontes de publicação. Também foi observado que as publicações estão mais concentradas em anos recentes, pois 24 dos 30 artigos identificados foram publicados de 2008 a 2012. Esta situação demonstra um interesse mais recente em analisar os fatores de impacto da modularidade em produto aos princípios do ecodesign e desenvolvimento de produtos sustentáveis. Além disso, grande parte desses trabalhos consiste de modelagem teórica de desenvolvimento de produtos considerando a modularidade e aspectos ambientais e maneira integrada, resultando em sistemáticas que consideram, além dos requisitos funcionais de produto, critérios como seleção de materiais menos tóxicos, reaproveitamento de componentes pós-uso, módulos multifuncionais, entre outros.

Verificou-se ainda que a maioria dos exemplos encontrados vem do setor automotivo, de eletrodomésticos e equipamentos eletroeletrônicos, em relação a outros setores industriais, sendo possível corroborar esses resultados com a literatura. Observou-se também que a modularidade, além de oferecer vantagens competitivas ao negócio, tais como economia de escala, aumento na viabilidade de mudanças em componentes/produtos, variedade de produtos oferecidos, facilidade 
de diagnóstico de falhas e defeitos e manutenção, também proporciona diferenciais quanto às variáveis ambientais, como o aumento da facilidade de utilização dos componentes ou materiais como recursos secundários, eficiente reciclagem, reuso e redução de perdas, além dos demais benefícios para os princípios e práticas do ecodesign, relatados neste trabalho.

Notou-se também que a predominância das práticas busca a melhor adequação do produto para a etapa de pós-uso, ou seja, destinação dos produtos após o seu consumo. A maioria das publicações visou a melhor disposição dos mesmos, por meio de desmontagem facilitada, reutilização de módulos, entre outras práticas relacionadas ao fim da vida útil dos produtos. Este fato denota em oportunidades para enfoque em práticas de modularidade/ecodesign no início do ciclo de vida dos produtos, com o intuito de desenvolver abordagens mais proativas.

Este trabalho é uma etapa de uma pesquisa em andamento, ou seja, os resultados e considerações apresentadas tem potenciais oportunidades de aprofundamento e direcionamento, conforme o foco de interesse. Além disso, o número reduzido de publicações encontradas no assunto pode ser explicado pelo fato de a modularidade ter uma abordagem mais voltada à perspectiva funcional e estrutural do produto do que do ponto de vista ambiental.

Os resultados obtidos mostram uma potencial contribuição teórica da estratégia de modularidade em produtos aos princípios, conceitos e práticas do ecodesign, uma vez que foi possível inferir benefícios e convergências na maioria das situações abordadas. Entretanto, há a necessidade de busca por resultados empíricos relacionados a essa convergência entre modularidade e ecodesign, com o intuito de se verificar como ocorrem as ações e contribuições práticas disso, para posterior comparação com a literatura acerca dos temas aqui analisados. Sugere-se então a ampliação do presente trabalho, analisando não somente a modularidade em produto, mas também as suas demais abordagens (produção e uso). Além disso, há a oportunidade de se aprofundar em determinados conceitos do ecodesign, de modo a melhor detalhar os benefícios, dificuldades e recomendações da aplicação da modularidade. 


\section{REFERÊNCIAS}

ALLY, J.; PRYOR, T. Accelerating hydrogen implementation by mass production of a hydrogen bus chassis. Renewable and Sustainable Energy Reviews, v. 13, n. 3, p. 616-624, 2009. http://dx.doi.org/10.1016/j.rser.2007.12.001

ALMEIDA, C.M.V.B. et al. Energy as a tool for ecodesign: evaluating materials selection for beverage packages in Brazil. Journal of Cleaner Production, v. 18, n. 1, p. 32-43, 2011.http://dx.doi.org/10.1016/j.jclepro.2009.03.019

ARNHEITER, E.D.; HARREN, H. A tipology to unleash the potential of modularity. Journal of Manufacturing Technology Management, v. 16, n. 7, p. 699-711, 2005. http://dx.doi.org/10.1108/17410380510619923

ARNHEITER, E.D.; HARREN, H. Quality management in a modular world. The TQM Magazine, v. 18, n. 1, p. 87-96, 2006.http://dx.doi.org/10.1108/09544780610637712

BALDWIN, C.Y.; CLARK, K.B. Managing in an age of modularity. Harvard Business Review, v. 75, n. 5, p. 84-93, 1997.

BALDWIN, C.Y.; CLARK, K.B. Design rules: the power of modularity. Cambridge: MIT Press, 2000.

BARBOSA, J.C.L. Eco-Design. In: CONGRESSO INTERNACIONAL DE PESQUISA EM DESIGN, 1 ,. e O CONGRESSO BRASILEIRO DE PESQUISA E DESENVOLVIMENTO EM DESIGN. 5., 2002. Anais do P\&D Design. Brasília, 2002.

BASK, A. et al. Modularity in logistics services: a business model and process review. International Journal of Services and Operations Management, v. 10, n. 4, p. 379399, 2011.http://dx.doi.org/10.1504/IJSOM.2011.043463

BCSD PORTUGAL - CONSELHO EMPRESARIAL PARA O DESENVOLVIMENTO SUSTENTÁVEL. Eco-eficiência. Disponível em: http://www.bcsdportugal.org/ecoeficiencia/102.htm. Acesso em: 29 mar. 2012.

BERTO, R.M.V.S.; NAKANO, D.N. A produção científica nos anais do encontro nacional de engenharia de produção: um levantamento de métodos e tipos de pesquisa.

Produção, v. 9, n. 2, p. 65-76, 2000.

http://dx.doi.org/10.1590/S0103-65131999000200005

BORCHARDT, M. ET AL. Adopting ecodesign practices: case study of a midsized automotive supplier. Environmental Quality Management, v. 19, n. 1, p. 7-22, 2009. http://dx.doi.org/10.1002/tqem.20232 
BYGGETH, S.; BROMAN, G.; ROBÈRT, K.H. A method for sustainable product development based on a modular system of guiding questions. Journal of Cleaner Production, v. 15, n. 1, p. 1-11, 2007.

http://dx.doi.org/10.1016/j.jclepro.2006.02.007

CARDOSO, M.A.; KISTMANN, V.B. Modularização e design na indústria automotiva: o caso do modelo fox da volkswagen do Brasil. Revista Produção Online, v. 8, n. 4, 2008.

CARIDI, M.; PERO, M.; SIANESI, A. Linking product modularity and innovativeness to supply chain management in the Italian furniture industry. International Journal of Production Economics, v. 136, n. 1, p. 207-217, 2012.

http://dx.doi.org/10.1016/j.ijpe.2011.11.012

CARNEVALLI, J.A.; VARANDAS JÚNIOR, A.; CAUCHICK MIGUEL, P.A. Uma investigação sobre os benefícios e dificuldades na adoção da modularidade em uma montadora de automóveis. Produto \& Produção, v. 12, n. 1, p. 60-90, 2011.

Carvalho, I. et al.. Reducing fuel consumption through modular vehicle architectures.

Applied Energy, v. 93, p. 556-563, 2012.

http://dx.doi.org/10.1016/i.apenergy.2011.12.004

CAUCHICK MIGUEL, P.A. Perspectivas e implicações da modularidade do desenvolvimento de produto - estudo bibliográfico inicial. SIMPÓSIO DE ENGENHARIA DE PRODUÇÃO, 11, 2004. Anais eletrônicos... Bauru-SP. Disponível em:

http://www.simpep.feb.unesp.br/anais_simpep_aux.php?e=11. Acesso em: 20 mar. 2012.

CAUCHICK MIGUEL, P.A.; CABRAL NETTO, O.V.; MARIOKA, S.N. Uma investigação sobre a adoção da modularidade no projeto de novos produtos e na produção em uma montadora automotiva. Produto \& Produção, v. 10, n. 3, p. 07-18, 2009.

CHRISTENSEN, T.B. Modularised eco-innovation in the auto industry. Journal of Cleaner Production, v. 19, n. 2-3, p. 212-220, 2011.

http://dx.doi.org/10.1016/j.jclepro.2010.09.015

Doran, D.et al. Supply chain modularisation: cases from the french automobile industry. International Journal of Production Economics, v. 106, n. 1, p. 2-11, 2007. http://dx.doi.org/10.1016/j.ijpe.2006.04.006

FERNANDEZ, I.; KEKÄLE, T. The influence of modularity and industry clockspeed on reverse logistics strategy: implications for the purchasing function. Journal of Purchasing and Supply Management, v. 11, n. 4, p. 193-205, 2005. http://dx.doi.org/10.1016/j.pursup.2006.01.005

Revista Produção Online, Florianópolis, SC, v.14, n. 2, p. 560-592, abr./jun. 2014. 
FIKSEL, J.R. Design for environment: creating eco-efficient products and processes. New York: McGraw-Hill. 1996.

FIRMO, A.C.C.; LIMA, R.S. Gerenciamento da cadeia de suprimentos no setor automobilístico: um estudo de caso no consórcio modular. Revista Produção Online, v. 5, n. 4, p. 866-873, 2005. http://dx.doi.org/10.14488/1676-1901.v5i4.381

FREDRIKSSON, P. Mechanisms and rationales for the coordination of a modular assembly system - the case of Volvo cars. International Journal of Operation and Production Management, v. 26, n. 4, p. 250-270, 2006.

http://dx.doi.org/10.1108/01443570610650530

GARCÍA, S.G. ET AL.. Eco-innovation of a wooden based modular social playground: application of LCA and DfE methodologies. Journal of Cleaner Production, v. 27, p. 21-31, 2012. http://dx.doi.org/10.1016/j.jclepro.2011.12.028

GEUM, Y.; KWAK, R.; PARK, Y. Modularizing services: A modified HoQ approach.

Computers \& Industrial Engineering, v. 62, n. 2, p. 579-590, 2012.

http://dx.doi.org/10.1016/i.cie.2011.11.006

Go, T.F.et al. Genetically optimised disassembly sequence for automotive component reuse. Expert Systems with Applications, v. 39, n. 5, p. 5409-5417, 2012.

http://dx.doi.org/10.1016/j.eswa.2011.11.044

GUNGOR, A.; GUPTA, S.M. Issues in environmentally conscious manufacturing and product recovery: a survey. Computers \& Industrial Engineering, v. 36, n. 4, p. 811853, 1999. http://dx.doi.org/10.1016/S0360-8352(99)00167-9

HUANG, C.C. ET AL. A novel approach to product modularity and product disassembly with the consideration of $3 R$-abilities. Computers \& Industrial Engineering, v. 62, n. 1, p. $96-107,2012$.

http://dx.doi.org/10.1016/j.cie.2011.08.021

Jacobs, M. et al. Product and process modularity's effects on manufacturing agility and firm growth performance. Journal of Production and Innovation Management, v. 28, n. 1, p. 123-137, 2011.

http://dx.doi.org/10.1111/j.1540-5885.2010.00785.x

JACOBS, M.; VICKERY, S.K.; DROGE, C. The effects of product modularity on competitive performance: Do integration strategies mediate the relationship?

International Journal of Operations \& Production Management, v. 27, n. 10, p. 10461068, 2007.

http://dx.doi.org/10.1108/01443570710820620

Revista Produção Online, Florianópolis, SC, v.14, n. 2, p. 560-592, abr./jun. 2014. 
KARLSSON, R.; LUTTROPP, C. EcoDesign: what's happening? An overview of the subject area of EcoDesign and of the papers in this special issue. Journal of Cleaner Production, v. 14, n. 15-16, p. 1291-1298, 2006.

http://dx.doi.org/10.1016/j.jclepro.2005.11.010

KUO, T.C. Enhancing disassembly and recycling planning using life-cycle analysis.

Robotics and Computer-Integrated Manufacturing, v. 22, n. 5, p. 420-428, 2006. http://dx.doi.org/10.1016/j.rcim.2005.11.014

LANGLOIS, R. Modularity in technology and organization. Journal of Economic Behavior \& Organization, v. 49, n. 1, p.19-37, 2002.

http://dx.doi.org/10.1016/S0167-2681(02)00056-2

LAU, A.K.W.; YAM, R.C.M.; TANG, E. The Impact of Product Modularity on New Product Performance: Mediation by Product Innovativeness. Journal of Product Innovation Management, v. 28, n. 2, p. 270-284, 2011.

http://dx.doi.org/10.1111/j.1540-5885.2011.00796.x

LAU, A.K.W.; YAM, R.C.M.; TANG, E. Factors influencing the relationship between product modularity and supply chain integration. International Journal of Operations \& Production Management, v. 30, n. 9, p. 951-977, 2010.

http://dx.doi.org/10.1108/01443571011075065

LAU, A.K.W.; YAM, R.C.M.; TANG, E. The complementarity of internal integration and product modularity: An empirical study of their interaction effect on competitive capabilities. Journal of Engineering and Technology Management, v. 26, n. 4, p. 305326, 2009. http://dx.doi.org/10.1016/i.jengtecman.2009.10.005

LIN, Y.; PEKKARINEN, S. QFD-based modular logistics service design. Journal of Business \& Industrial Marketing, v. 26, n. 5, p. 344-356, 2011.

http://dx.doi.org/10.1108/08858621111144406

LORENZI, S.; LELLO, A.D. Product modularity theory and practice: the benefits and difficulties in implementation within a company. International Journal of Automotive Technology and Management, v. 1, n. 4, p. 425-448, 2001.

http://dx.doi.org/10.1504/IJATM.2001.000050

LUTTROPP, C.; LAGERSTEDT, J. EcoDesign and the ten golden rules: generic advice for merging environmental aspects into product development. Journal of Cleaner Production, v. 14, n. 15-16, p. 1396-1408, 2006.

http://dx.doi.org/10.1016/j.jclepro.2005.11.022

Revista Produção Online, Florianópolis, SC, v.14, n. 2, p. 560-592, abr./jun. 2014. 
MACHADO, A.G.C.; MORAES, W.F.A. Estratégias de customização em massa implementadas por empresas brasileiras. Produção, v. 18, n. 1, p. 170-183, 2008. http://dx.doi.org/10.1590/S0103-65132008000100013

MIKKOLA, J.H.; GASSMANN, O. Managing modularity of product architectures: toward an integrated theory. IEEE Transactions on Engineering Management, v. 50, n. 2, p. 204-218, 2003. http://dx.doi.org/10.1109/TEM.2003.810826

MORRIS, D.; DONELLY, T.; DONELLY, T. Insights from industry: supplier parks in the automotive industry. Supply Chain Management: An International Journal, v. 9, n. 2, p. 129-133, 2004. http://dx.doi.org/10.1108/13598540410527024

MURPHY, T. Brake force, with advics brake market may never be the same. Ward's Auto World, v. 38, n. 4, p. 42, 2002.

NUNES, B.; BENNETT, D. The contribution of modularity to green operations practices. Brazilian Journal of Operations \& Production Management, v. 5, n. 2, p. 93-108, 2008.

ORSATO, R.; WELLS, P. Introduction - the automobile industry \& sustainability. Journal of Cleaner Production, v. 15, n. 11-12, p. 989-993, 2007.

http://dx.doi.org/10.1016/i.jclepro.2006.05.035

Pandremenos, J.et al. G. Modularity concepts for the automotive industry: a critical review. CIRP Journal of Manufacturing Science and Technology, v. 1, n. 3, p. 148152, 2009.http://dx.doi.org/10.1016/j.cirpj.2008.09.012

PARENTE, R.C.; BAACK, D.W.; HAHN, E.D. The effect of supply chain integration, modular production, and cultural distance on new product development: A dynamic capabilities approach. Journal of International Management, v. 17, n. 4, p. 278-290, 2011. http://dx.doi.org/10.1016/j.intman.2011.08.001

PERSSON, M. Effects of changing a module's interface: a case study in an automotive company. International Journal of Automotive Technology Management, v. 6, n. 3, p. 331-345, 2006. http://dx.doi.org/10.1504/IJATM.2006.012124

RAMANI, K.et al. Integrated sustainable life cycle design: a review. Journal of Mechanical Design, v. 132, n. 9, p. 1-15, 2010.

http://dx.doi.org/10.1115/1.4002308

RODRIGUES, E.A.; CARNEVALLI, J.A.; CAUCHICK MIGUEL, P.A. Aplicação da modularidade no projeto do produto e na produção: uma análise em uma montadora de caminhões e ônibus. GEPROS - Gestão da Produção, Operações e Sistemas, v. 4, n. 4, p. 79-92, 2009.

Revista Produção Online, Florianópolis, SC, v.14, n. 2, p. 560-592, abr./jun. 2014. 
SALERNO, M.S.; CAMARGO, O.S.; LEMOS, M.B. Modularity ten years after: an evaluation of the Brazilian experience. International Journal of Automotive Technology and Management, v. 8, n. 4, p. 373-381, 2008.

http://dx.doi.org/10.1504/IJATM.2008.020309

SALERNO, M.S. et al. The importance of locally commanded design for the consolidation of local supply chain: the concept of design headquarters. International Journal of Manufacturing and Management, v. 16, n. 4, p. 361-376, 2009.

SILVA, H.O.L.; CAUCHICK MIGUEL, P.A. Adoção da modularidade no desenvolvimento de produto - um estudo de caso em uma montadora de veículos. SIMPÓSIO DE ENGENHARIA DE PRODUÇÃO, 13, 2006. Anais eletrônicos... Bauru-SP. Disponível em: http://www.simpep.feb.unesp.br/anais_simpep.php?e=4. Acesso em: 02 abr. 2012.

SUSTAINABLE JERSEY. A better tomorrow, one community at a time. 2010.

Disponível em: http://my.communityplaces.org/newnjssi/editor/doc/p14!121.pdf. Acesso em: 02 mai. 2012.

TOYOTA. Toyota Prius. Disponível em:

http://www.toyota.pt/cars/new_cars/prius/index.tmex. Acesso em: 02 mai. 2012.

VENZKE, C. A situação do ecodesign em empresas moveleiras da Região de Bento Gonçalves-RS: Análise das posturas e práticas ambientais. Dissertação (Mestrado em Administração). Universidade Federal do Rio Grande do Sul (UFRGS), Porto Alegre-RS. 2002.

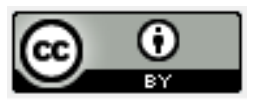

Artigo recebido em 21/08/2012 e aceito para publicação em 01/11/2012

DOI: http://dx.doi.org/10.14488/1676-1901.v14i2.1424 